\title{
Martian Surface Composition as Determined by the MGS Thermal Emission Spectrometer
}

Steve R. Ruff, Philip R. Christensen, Joshua L. Bandfield, Victoria E. Hamilton

Arizona State University, Tempe, AZ 85287-1504, USA

Hugh H. Kieffer

U.S. Geological Survey, Flagstaff, AZ 86001, USA

Richard V. Morris, Melissa D. Lane

NASA Johnson Space Center, Houston, TX 77058, USA

Michael C. Malin

Malin Space Science Systems, San Diego, CA 92191-0148, USA

Abstract. The surface composition of Mars has been investigated using the Thermal Emission Spectrometer (TES) instrument during the mapping phase of the Mars Global Surveyor mission. The TES has mapped $\sim 85 \%$ of the Martian surface at a resolution of 3-9 km. Separation of the atmospheric dust, water-ice cloud, $\mathrm{CO}_{2}$, water vapor, and surface components has been accomplished using radiative transfer and deconvolution. Two distinct surface compositional units have been mapped; (1) a basalt with plagioclase feldspar, Ca-rich pyroxene, minor sheet silicates; and (2) a basaltic andesite with silica glass, plagioclase, and minor pyroxene. Three large-scale (100's $\mathrm{km}$ ) accumulations of hematite have been found in Sinus Meridiani, Aram Chaos and Ophir/Candor Chasms. These regions are interpreted to be formed by aqueous precipitation under either ambient or hydrothermal conditions. No surfaces with detectable abundances of carbonate have been found. The albedo of the surface has been mapped with an absolute accuracy of $\sim 1-2 \%$ and significant changes in surface albedo have occurred from the orbital measurements obtained by the Viking IRTM instrument. 